\title{
Accelerometer Performance for Maker Faire Quadrotor with Kalman Filtering Using PID Controller
}

\author{
Muhammad Zaim ${ }^{1, a^{*}}$, Elmi Abu Bakar ${ }^{1, b}$, Low Hock Soon ${ }^{1, c}$ \\ ${ }^{1}$ School of Aerospace Engineering, Universiti Sains Malaysia, Engineering Campus, 14300 Nibong \\ Tebal, Pulau Pinang, Malaysia \\ amuhdzaim90@gmail.com, ${ }^{\mathrm{b}}$ meelmi@usm.my, chocksoon@gmail.com
}

Keywords: Kalman filter, quadrotor, PID controller

\begin{abstract}
This paper presents a study on quadrotor using PID controller together with the application of Kalman Filter. Purpose of this project is to study effect of separate Kalman filter in overcoming the signal noise and gyro drift in the attitude sensors and simulate the PID controller which controlling the quadrotor dynamics through damping the vehicle oscillation. In this research, simulation of Kalman Filter in filtering the noise from the inertial moment unit (IMU) and PID controller in damping on unstable oscillation has been conducted to observe the performance of the quadrotor.
\end{abstract}

\section{Introduction}

Quadrotor is a special variant of air vehicle having abilities of lifting, landing, and vertical take-off. Several inventions have been done in forming this quadrotor starting with the first studies on rotorcraft were pioneered by Leonardo da Vinci in 1493. Control was to be provided by cables that could alter the pitch of the rotor blades, and by steerable vanes at either end of the machine intended to direct the downwash of the rotors.

Quadrotor can be controlled by only changing the speed of each rotor. Since, a quadrotor can be controlled by only changing the motor speed, it has much simpler mechanical structure and easier to be controlled compared to helicopter. For a quadrotor to have a stable flight, it must have a closed loop feedback system by regularly monitor the quadrotor attitude to control the flying dynamics. In order to implement the closed loop feedback control system to the quadrotor plant, noises from the attitude sensors are inevitable. Therefore, complementary filter and Kalman filter will be reviewed for their application. Besides, flight dynamics and stability especially the PID controller will also be covered because there is the need to damp the oscillation caused by the dynamic behavior and external disturbance on the quadrotor.

A robust PID control strategy, tuned via affine parameterization, has been presented. Equations used in the simulation model are coupled, whereas the equations used for control are decoupled. It can be seen how the behavior of the system is quite good even with the simplifications made. So a nonlinear system can be controlled with a decoupled linear controller. This controller is a PID tuning by affine parameterizations and robust thanks to $H_{\infty}$ theory (Garcia et al., 2012).

To control the position of the quadrotor, a PD controller for each axis is created independently. The controllers separately for the position and angle are developed then are put together in one model. It was necessary to add a fast pole to make our control system more realistic because the order of the numerator was higher than the denominator. The parameter found is input using the independent controllers (Miller, 2008). 


\section{Methodology}

\subsection{Quadrotor Modeling}

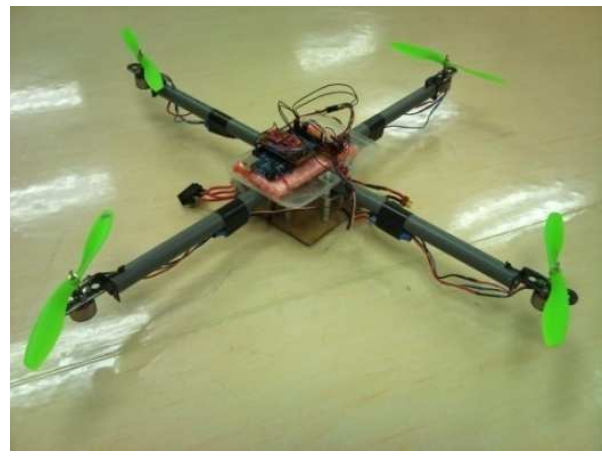

Figure 1: Maker Faire Quadrotor

This quadrotor is using the ' + ' configuration and consisting of four propellers in total. Two spinning in the clockwise $(\mathrm{CW})$ direction while the other two in the counter-clockwise $(\mathrm{CCW})$ direction. Quadrotor is an under-actuated system which means with only four actuator (motor/propeller), it is able to control all six degree of freedom (DOF). This under-actuated implies that two DOF, the translational motion in the $\mathrm{x}$ and $\mathrm{y}$ direction is coupled with the pitch and roll rotational motion respectively. By allowing two of the propellers spinning in the $\mathrm{CW}$ direction and the other two in the $\mathrm{CCW}$ direction cancel off the torque in the yaw direction and thus eliminate the need of additional anti-torque actuator. With the condition both $\mathrm{CW}$ and $\mathrm{CCW}$ propellers are producing equal amount of thrust.

Table 1: Quadrotor Thrust Configuration

Motion
All four propellers produce the similar amount of thrust and
hover at certain altitude. By reducing or increasing the
similar amount of thrust on each propeller, quadrotor altitude
will be lower or higher accordingly.
Yaw Right
By maintaining the rotational speed of CW propellers and
reducing the CCW, a net torque will be induced in the yaw
direction. In this case, yaw to the right.

\subsection{Control Algorithm}

Inertial Moment Unit consists of accelerometer and rate gyros continuously inform the microcontroller whether the vehicle is in a stable attitude. If it is not in the stable attitude, the controller unit will process and calculate the suitable thrust and speed by powering the electronic 
speed controller and propeller. The thrust produced will return the vehicle to the stable attitude. Meanwhile, user is able to insert desired input by moving the control stick on the radio frequency transmitter which transmits the signal to the radio frequency receiver. Fig. 3 summarizes the communication relationship between electronic components within the quadrotor.

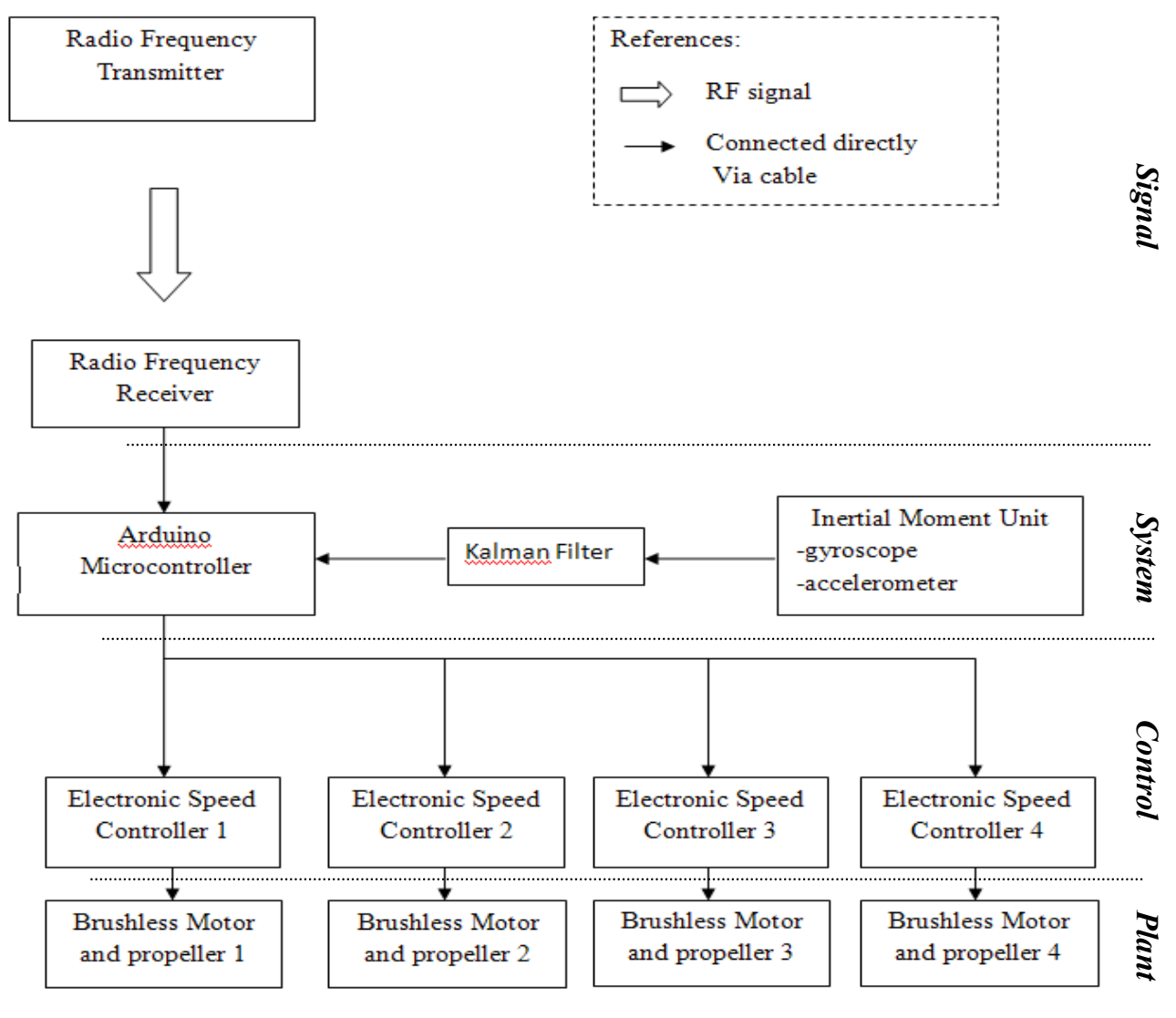

Figure 3: Relationship among electronic components

This is a closed loop feedback control system, which means there is a measurement device that frequently measures the instantaneous state and report to the controller. The sensors is considered perfect therefore is regarded as unity feedback. The subsystem consists of the control input which is the desired roll or pitch angle, PID controller and quadrotor dynamic plant.

\section{Result and Discussion}

\subsection{Simulation of Kalman Filter on Accelerometer Signal}

The raw angle read from an accelerometer while the angular velocity obtained from a rate gyro. As can be seen from Fig. 4, there, both of the reading from the sensors contains a lot of noise. When come to the attitude determination of a flying quadrotor, a smooth and reduced noise reading would be helpful in maintaining the stability of the quadrotor. Initially, the IMU which contain both the accelerometer and rate gyro is placed on a horizontal place. It is then rotated clockwise along the $\mathrm{x}-$ axis direction, this motion cause a positive deviation in both sensors from $t=4 s$ to $t=5 s$. This position is maintained for 5 seconds. Afterward, the IMU is rotated counterclockwise until it is horizontally aligned, this motion shows the negative deviation from $t=10 \mathrm{~s}$ to $t=11 \mathrm{~s}$. 




Figure 4: Raw Angle and Angular Velocity Response

By taking Kalman filter algorithm, state model noise and measurement model noise is tuned until the Kalman filter angle response in the simulation show much reduced noise level as displayed in Fig. 5. Both of the raw angle and angular velocity reading obtained from the sensors are filtered to obtain a smoother and reduced noise angle. As can be seen in Fig. 5, raw angle from the accelerometer is represented by the green curve while the blue curve is the filtered angle. Filtered angle shows a contrast reduced in the noise level from the accelerometer angle. Besides, filtered angle has no time lagging behind the accelerometer angle, which indicates the response rate is in line with the real motion.

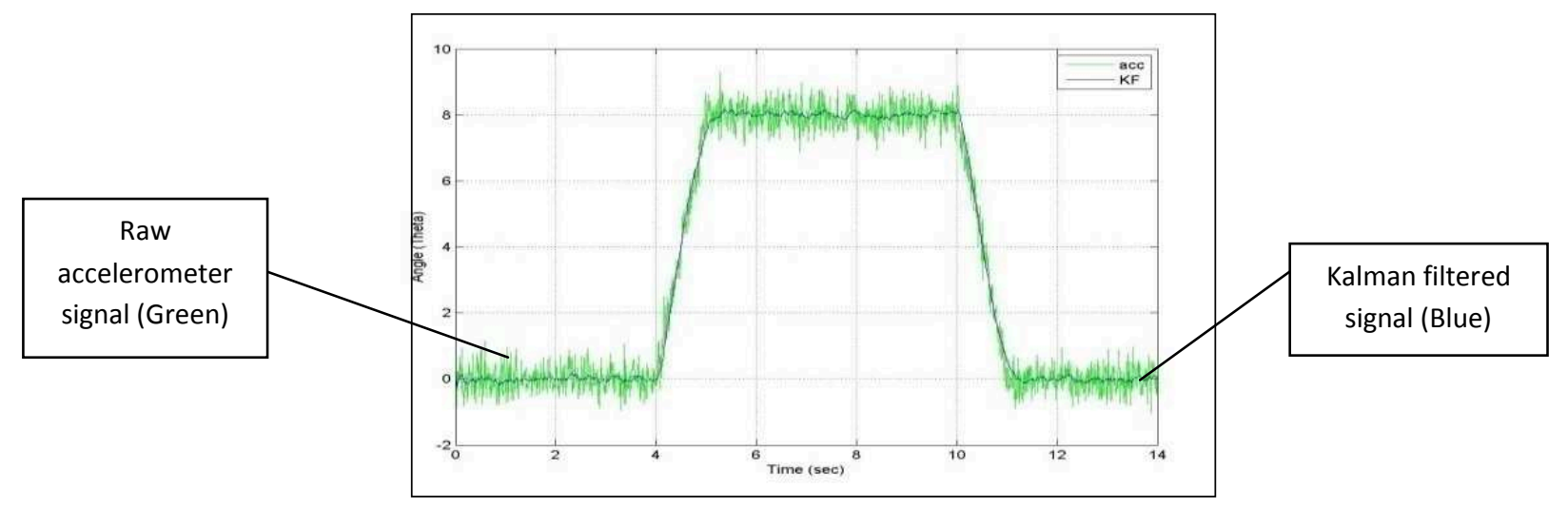

Figure 5: Filtered Angle Response of Accelerometer Signal

\subsection{Implementation on Quadrotor}

\subsubsection{Kalman Filter}

\subsubsection{Pitch Angle}

By having a PD controller in controlling the pitching moment with proportional term tuned to 1.4 while derivative term with value 0.5 , with the similar tuning value of state model noise and measurement model noise as shown in the simulation part, in figure 7 the hardware's Kalman filter response does not resemble the simulation's Kalman filter response and there is lagging behind the accelerometer reading. 


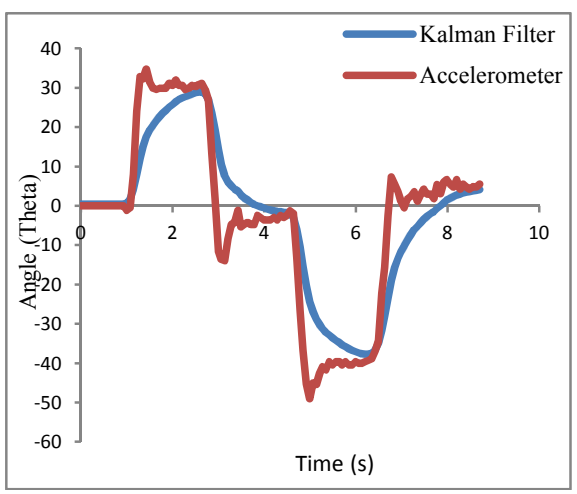

a)

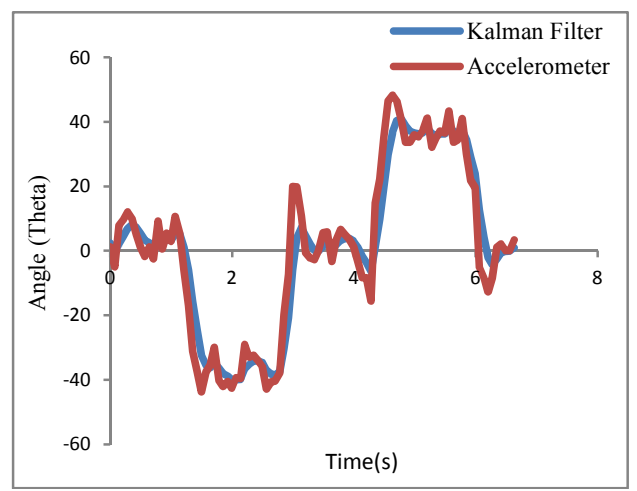

b)

Figure 7: Pitch Angle Response a) without motor spinning b) with motor spinning.

\subsubsection{Roll Angle}

Similarly to the pitch angle response, figure 8 shows the roll angle response using the theoretical tuning value of state and measurement model noise used in the simulation. The Kalman response shows time lagging behind the accelerometer reading which indicates the slow response.

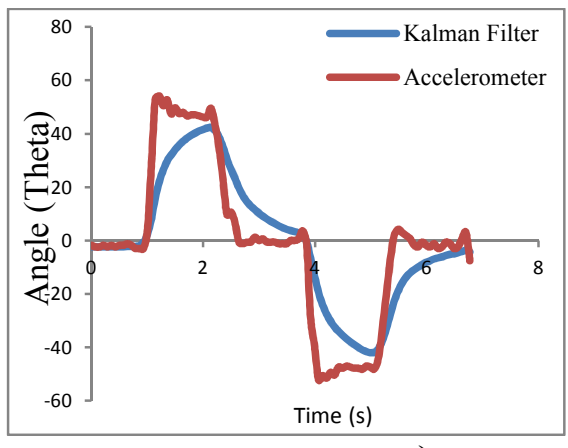

a)

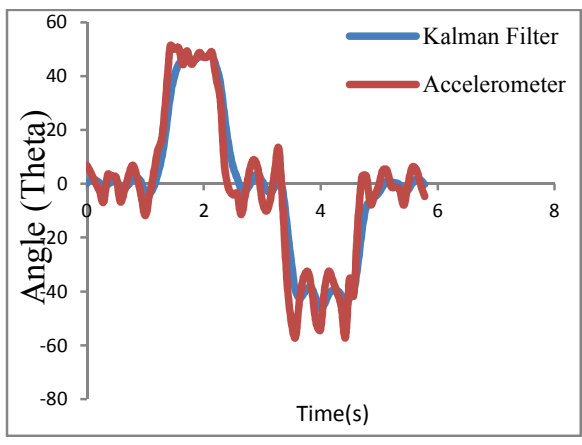

b)

Figure 8: Roll Angle Response a) without motor spinning b) with motor spinning.

\section{Conclusions}

Kalman Filter is useful in reducing the noise level in the reading measured from the accelerometer and rate gyro as well as increasing its accuracy. Result obtained with reduced noise level is beneficial since the quadrotor requires high precision and quick angle response in order to maintain the vehicle in a stable state. Individual filter for roll and pitch angle and also simplification made in the Kalman filter by introducing as much zero in the matrix cell has reduced the complexity in computing the filtered angle. The result obtained in the simulation and the hardware differs greatly, this is due to the simplification made on the Kalman filter algorithm and the matrix.

Proportional and derivative, PD controller is implemented in the simulation instead of PID controller. This control alone is able to produce a good result. Both the roll and pitch angle response is critically damped but at different time with the aid of Simulink. 


\section{REFERENCES}

[1] Atheer L. Salih, M. Moghavvemi, Haider A. F. Mohamed and KhalafSallomGaeid, (2010)“Modeling and PID Controller Design for a Quadrotor Unmanned Air Vehicle”.

[2] Bouabdallah S. and Siegwart R., (2007 ) "Full Control of a Quadrotor".

[3] Bouabdallah S., "Design and control of quadrotors with application to autonomous flying", Ph.D. dissertation, EPFL, 2006.

[4] Gabriel M. Hoffmann, Haomiao Huang, Steven L. Waslander and Claire J. Tomlin, (2011) "Precision flight control for a multi-vehicle quadrotor helicopter testbed".

[5] Gabriel M. Hoffmann,Huang H., Steven L. Waslander and Claire J. Tomlin,(2007) "Quadrotor Helicopter Flight Dynamics and Control: Theory and Experiment", American Institute of Aeronautics and Astronautics.

[6] GarciaR.A., RubioF.R. and OrtegaM.G., (2012) "Robust PID Control of the Quadrotor Helicopter".

[7] LeffertsE.J., MarkleyF. L., ShusterM. D., "Kalman filtering for spacecraft attitude estimation", Journal of Guidance, Control and Dynamics 5 (1982) 417-429

[8] Miller K., (2008) "Path Tracking Control for Quadrotor Helicopter".

[9] Nicol C., MacnabC.J.B. and Ramirez-Serrano A., (2011) "Robust adaptive control of a quadrotor helicopter".

[10] Xiong K., Liang T. and Yongjun L., (2010) "Multiple modelKalman filter for attitude determination of precision pointing spacecraft". 